\title{
SCIDOC
}

\author{
International Journal of Dentistry and Oral Science (IJDOS) \\ ISSN: 2377-8075
}

\section{The Diagnostic Role of P16ink4a in Detecting High-Risk HPV16 in a Group of Syrian Nasopharyngeal Carcinoma Patients}

Rasha Ali Mohammad ${ }^{1 *}$, Ahmad Al Manadili1 ${ }^{1}$

${ }^{1}$ Department of Oral Histology and Pathology, Faculty of Dentistry, Damascus University, Damascus, Syria.

\section{Abstract}

Introduction: P16ink4a is a tumor suppressor gene, and it plays a critical role in cell cycle organizing and aging through the regulation of the Cyclin-Dependent Kinase (CDK) 4/6 and Cyclin D complexes. its inactivation corresponds with human carcinogenesis. The overexpression of $\mathrm{p} 16$ is considered to be a useful marker to investigate human papilloma virus (HPV) in certain cancers, but its role in nasopharyngeal cancer patients' outcomes is still unclear.

Objectives: We aimed in this study to detectHPV high risk type16 expression in a sample of 89 nasopharyngeal carcinoma Syrian patients, and to detect expression of P16ink4a in the same sample. After that, we studied the correlation between the two expressions of HPV and P16ink4a and the power ofcorrelation if existed.

Materials and Methods: Eighty n patients with nasopharyngeal carcinoma (NPC) treated in al Moasat universal hospital between 2010 to 2017 were chosen. To investigate the expression of P16ink4aand high-riskHPV 16 (HPVhr 16), we used immunohistochemistry staining. We evaluate the diagnostic role of p16 by discussing the expression profile of p16 in a group of NPC Syrian patients, especially those with HPVhr 16 positive expression .

Results: The results of this study showed that the ratio of HPVhr 16 positive expression in 89 patients was $13.5 \%$, and P16ink 4 a positive expression was $15.7 \%$, and there was a positive, good correlation between expression of both HPVhr and P16ink4a, so it could be an alternative aid to detect HPVhr16 in paraffin embedded formalin sections. Nonetheless more studies are needed to evaluate the importance of the lack of HPVhr 16 and P16ink4aexpression when correlate with stages of the cancer and other clinical data.

Conclusions: The results of this study showed thatratio of HPVhr 16expression is $13.5 \%$ and it is considered a weak percentage but it motivate other studies to assure the good prognosis of patients with no virus existence .Expression of P16ink4a is 15.7\% and it can be a surrogate marker for HPVhr 16 in the sample of 89 NPC patients. More studies are needed to correlate prognosis with presence of P16ink4a.

Keywords: Human Papilloma Virus; Nasopharyngeal Carcinoma; P16ink4a; High Risk.

\section{Introduction}

Nasopharyngeal carcinoma(NPC) is an uncommon tumor that arises in the epithelium of the nasopharynx [1]. The wide difference in the incidence among different people and geographic areas suggests a considerable association of NPC with diverse genetic and environmental factors [2]. Its etiology is not specified enough, because of multiple complex factors that affect its emergence. One of the obstacles that make its cure harder, is its late diagnosis, because of the similarities between the presenting symptoms and other diseases [1].
Lately, there was a huge evidence of a certain involvement and a significant pathogenic role of HPV in the occurrence of oropharyngeal carcinoma, but still little is known about the prevalence and epidemiological role of this virus in NPC and other sites of head and neck sites $[3,4,9]$. Furthermore, HPV has been recently proposed to have a suspicious role in the etiology of non-endemic nasopharyngeal carcinoma [5-7]. On the contrary, a Belgium study reported that, the high incidence of positive HighRisk human-papilloma virus in oral cancers, the very worse outcomes for these tumors when it's compared with negative HPV in oral cavity cancers [8].

\section{*Corresponding Author}

Rasha Ali Mohammad DDS-MSc,

Department of Oral Histology and Pathology, Faculty of Dentistry, Damascus University, Damascus, Syria.

Tel: +963932341263

E-mail: rashasep@yahoo.com

Received: November 11, 2020

Accepted: December 28, 2020

Published: January 08, 2021

Citation: Rasha Ali Mohammad, Ahmad Al Manadili. The Diagnostic Role of P16ink4a in Detecting High-Risk HPV16 in a Group of Syrian Nasopharyngeal Carcinoma Patients. Int J Dentistry Oral Sci. 2021;8(1):1302-1307. doi: http://dx.doi.org/10.19070/2377-8075-21000258

Copyright: Rasha Ali Mohammad ${ }^{\circ}$ 2021. This is an open-access article distributed under the terms of the Creative Commons Attribution License, which permits unrestricted use, distribution and reproduction in any medium, provided the original author and source are credited. 
In the general, there are no approved criteria that are accepted all over the world about the preferable standard for High-Risk HPV in formalin-fixed, paraffin- embedded sections, nor a sufficient vision about the role of this High-Risk virus in locations different from the oropharynx [9].

The p16 gene, an important cell cycleregulator for G1 restriction checkpoint [10], is inactivated ina remarkable ratioof primary tumors. The absence of p16 may result in Retinoblastoma phosphorylation and uninhibited proliferation of NPC cells [11]. Despite the predictive role of p16 when it's overexpressed as a surrogate for human-papilloma virusin oropharyngeal carcinoma, it's role is notcertain in nasopharyngeal carcinomaas a prognosticator biomarker [12], and that is consistent with the biological nature of $\mathrm{p} 16$ whose expression is ascended as a direct reaction to uncontrolled proliferation [13].

Using p16 as a predictive marker to investigate risk factors in nasopharyngeal carcinoma may confront plenty of challenges when immunohistochemical staining $[14,15]$, for example the criteria that determine when $\mathrm{p} 16$ is positive , and when it is overexpressed $[5,6,16]$.

Our study aimed to detect the presence of human papilloma virus in a group of Syrian patients having nasopharyngeal carcinoma and to ensure the correlation between p16 expression and HighRiskHPV virus existence as it is not certain to be used as a surrogate marker of this virus in nasopharyngeal carcinoma.

\section{Objectives}

$\checkmark$ To detect HPVhr 16 expression in a sample of 89 patients of nasopharyngeal carcinoma.

$\checkmark$ To detect P16ink4a expression in the same sample

$\checkmark$ To compare the expression of HPVhr 16with expression of P16ink4a to discuss its diagnostic role.

\section{Materials and Methods}

\section{Patients specimens}

The number of samples collected from the archive of Al Moasat university hospital between the years 2010-2017 was 94 samples of nasopharyngeal carcinoma. First, collecting samples was based on the availability of both data of the patients and paraffin blocks related to his/her case. We revised the H\&E chosen slides to ensure the nasopharyngeal carcinoma diagnosis. we exclude the cases that lack data, and/or paraffin blocks, so they were 89 samples after excluding five cases.

\section{Immunohistochemical staining}

The immunohistochemical stain for P16ink4a using Anti-p161nk4a (CDKN2A) antibody, and HPVhr 16 using a mouse monoclonal antibody, from BioSB was performed using BioSB histology kit.we began the staining with deparaffinization by incubation the slides for one hour at $60^{\circ} \mathrm{C}$ degrees, then changing of xylene and absolute alcohol 3 to 2 changes until we put them in water, after that the step of the antigen retrieval by boiling in microwave for 30 min, later we wash the sides with Distilled Water(DW) two to three times, then blocking by incubate sections at room tem- perature with hydrogen peroxidase for 10 minutes, then incubate in Tris Buffered Saline TBS for five minutes. Adding the primary antibody (p16 or HPV) for about an hour, then washing slides with TBS, after that incubate for $10 \mathrm{~min}$. addingHRP for $45 \mathrm{~min}$ to one hour, after that we wash slides with TBS 3-5 times, and incubate for $10 \mathrm{~min}$, then adding $250 \mathrm{ul}$ of $1 \%$ di-amino-benzidine, later we wash the slides with DW 3-5 times, Finally counterstaining with hematoxylin for $1 \mathrm{~min}$.

\section{P16ink4a antibody Immunohistochemical staining}

We used Anti-p161nk4a (CDKN2A) antibody, Rabbit monoclonal, RM267 monoclonal from Sigma-Aldrich, reacts to human P16ink4a (Cyclin-dependent kinase inhibitor 2A). The protocol of staining was performed according to instructions supplied by the kit of BIO SB company. we used 1:1000 dilution after plenty of trials to conceive the appropriate staining. We used formalin -fixed and paraffin-embedded human colon cancer sections as control samples.

\section{HPVhr 16 immunohistochemical staining}

HPV 16 we used is a mouse monoclonal antibody that is concentrated, dialyzed, filter sterilized and diluted in buffer pPH7.5. its isotype is IgG2a and had nuclear localization. according to instructions of manufacturing company BIO SB, we handled the samples with the HPVhr 16 antibody as steps mentioned in immunohistochemical staining previously, and we used oral papilloma sections as positive control samples.

\section{Criteria of staining}

For P16ink4a staining Every sample was given a score that indicate the intensity of nucleus or cytoplasmic staining (no staining $=0$, weak staining $=1$, moderate staining $=2$, strong staining $=3$ ), and the extension of staining is measured also by numbers cells $(0 \%=0,1-10 \%=1,11-50 \%=2,51-80 \%=3,81-100 \%=4$. We determined the final results by multiplying the intensity scores with the extent of positivity scores, so the final score we get is limited between 0 as the minimum score and 12 as a maximum score [17-19].

For HPVhr 16 antibody staining, every slide has a nuclear staining (weak and /or strong) regarded positive.

\section{Statistical Analysis}

The results we get were subjected to statistical analysis using the Chi-Square Independence Test with ( $\mathrm{p}$-value $<0.05$ ), Spearman correlation, and SPSS version 25 statistical program for analyzing data.

\section{Results}

\section{Expression of HPVhr 16 and P16ink4a in NPC sections}

We found that 12 cases of 89 were HPVhr 16 positive which means $13.5 \%$ of all cases studied, whereas 14 cases of 89 had positive expression of P16ink4a, which means about $15 \%$ of all cases studied, and the positivity was ranged between high, low and no expression, (Table 1) depending on the result of multiplying 
density score by intensity one [17-19].

We can notice the pale nucleus staining and strong staining very obviously of HPVhr16 in (Figure 1), and the obvious brown staining of nucleus in basal layers only, (Figure 2). which both show the different expression appearance of HPVhr16 in immunohistochemical staining.

Whereas staining of P16ink4a is seen either in the whole epithelium adjacent to NPC lesion, (Figure 3).

Relation between HPVhr16 expression and P16ink4a staining

When we compared the percentage of positive expression of HPV and P16ink4a, we found that $50 \%$ of NPC patients who had positive expression of HPVhr 16, expressed positivity of P16ink4a, but patients who didn't express positivity of the virus, had positive expression of P16ink4a in $50 \%$ of cases also.

Only $9.9 \%$ of NPC patients who have the infection of HPVhr 16 expressed negative expression of P16ink4a, which means that expression of p16 didn't give the purpose it was used for in these limitedcases, whereas $90.1 \%$ of NPC patients included in this study showed negative expression of both P16ink4a and HPV (Table 2)

\section{Discussion}

HPVHR 16 Expression in nasopharyngeal carcinoma

Since HPVhuman papilloma virus is involved in the etiology of

Table 1. Expression of P16ink4a detected in 89 patients of NPC.

\begin{tabular}{|c|c|c|}
\hline Percentage & Frequency & Expression \\
\hline $9.0 \%$ & 8 & High \\
\hline $6.7 \%$ & 6 & Low \\
\hline $84.30 \%$ & 75 & Non \\
\hline
\end{tabular}

Table 2. HPV expression *P16ink4a expression in 89 patients of NPC ur.

\begin{tabular}{|c|c|c|c|c|}
\hline \multicolumn{2}{|c|}{$\begin{array}{c}\text { Negative expression } \\
\text { (no expression) }\end{array}$} & \multicolumn{2}{|c|}{$\begin{array}{c}\text { Positive expression } \\
\text { (high /low) }\end{array}$} & P16ink4a \\
\hline HPV- & HPV+ & HPV- & HPV+ & expressionHPV \\
\hline 73 & 8 & 4 & 4 & frequency \\
\hline $90.10 \%$ & $9.90 \%$ & $50 \%$ & $50 \%$ & percentage \\
\hline
\end{tabular}

Figure 1. The localized staining of HPVhr16 antibody in the epithelium of NPC section, magnification $\times 40$.

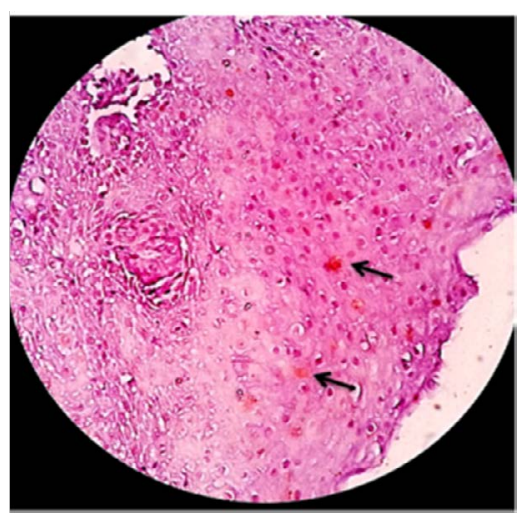

Figure 2. Immunohistochemical Staining of HPVhr16, we can notice the nucleus staining is only obvious in the basal layers of epithelium peripheral to NPC lesion. Magnification $\times 10$.

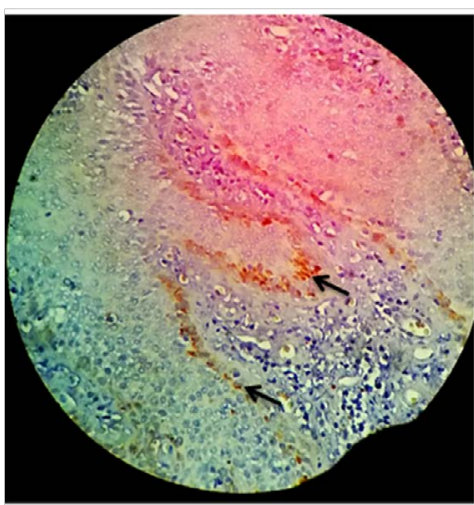


Figure 3. Expression of P16ink4a, notice the staining in the whole epithelium layers and in both nucleus and cytoplasm of cells in the epithelium peripheral to the lesion, while no staining in the connective tissue.

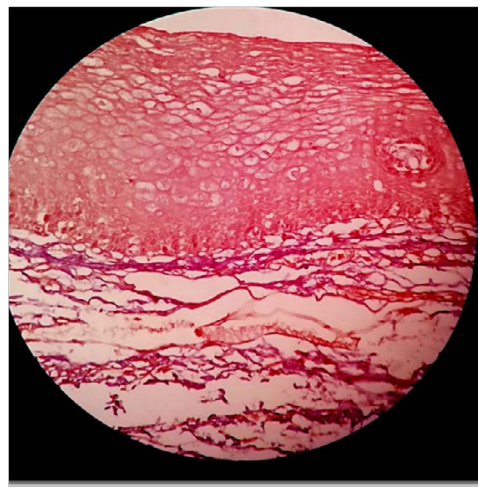

many head and neck cancers, they found that particularly oncogenic HPV subtype 16, has been accused to be the primary etiologic agent in squamous cell carcinoma cancers related to head and neck region, and they exhibit improved prognosis compared to patients with HPV negative tumors $[20,21]$.

When we investigate the HPV virus in nasopharyngeal carcinoma, we chose the high risk type (16) to be discussed and Since there are no universally accepted gold standard for High-RiskHPV assessment in formalin-fixed paraffin-embedded tissue sections [9], we followed standard in previous studies which used IHC staining to detect the virus.

HPV expression was positive in $13.5 \%$ of 85 patients of NPC, and that is less than the ratio documented in other studies like Walline and colleagues which was 5o\% [9]. Also, in another study they had found the expression is $30 \%$ HPV positive, and that could be explained because of the variable methods used to detect the virus, and the differences in the study design, if the samples were collected with most possibility of having HPV infection from the beginning, or they detect the virus after collecting the sample of nasopharyngeal carcinoma patients. in a random way [22].

Because its high sensitivity for HPV, we could assert that P16ink4a a surrogate marker for HPVhr 16 in NPC and that resultis compatible with other studies like (Walline, Komarck et al., 2013, on the other hand it was not the same conclusion in studies like Bonomi and his colleagues' study [23], and our explanation is because of various reasons, like size of sample, criteria of expression and variety of methodology used in detecting both the P16ink4a marker and HPVHR 16 virus.

\section{P16ink4a expression in Nasopharyngeal Carcinoma in gen-} eral

The expression of p16 had been evaluated in many studies of nasopharyngeal carcinoma, and reported to be reduced in expression and average of reduction ranges between $40-82 \%[13,24$, $25]$, which is compatible with our study that shows absence ofexpression of P16ink $4 \mathrm{a}$ in $84 \%$ of cases studied, as it is shown in table (1).

Absent of P16ink4al correlate with poor clinical outcome, and it could be involved in NPC development or progression but mechanism is still needs explanation, on the other hand, p16 is frequently deleted, mutated or methylated in several head and neck squamous cell carcinoma $[26,27,13]$.

\section{Expression of P16ink4a in 89 NPC Patients}

The percentage of expression of P16ink4a was (15.7\%), and it was similar to a UK study that showed $16 \%$ over expression [3], our percentage has gathered both low and high expression,but the UK study took the over expression of P16ink4a only. We can explain that result depending on previous studies showed that the loss of P16ink4a is increasingly common with advancing stages of various neoplasms, and in fresh non cultured primary tumors, specially that we didn't classified the samples depending on tumors' stages because of lack of clinical information and difficulties of proceeding each case after treatment.

when P16ink4a has positive expression in $50 \%$ of cases of NPC patients, there was no HPV infection detected. This result is congruent with a specific study of sinonasal undifferentiated carcinoma [28], which found overexpression of p16 in the absence of HPV expression.

We found that about $90 \%$ of cases that showed negative HPV, had also a negative expression of P16ink4a, as it is mentioned in the study of Veganzones and his colleagues, who explained the result because of loss of heterozygosity, and DNA hyper-methylation of the gene. Other researches documented that, about $90 \%$ of HPV-negative HNSCC tumors, exhibit low expression of P16ink4a, and it is compatible with our result $[29,30]$.

We found also $9.9 \%$ of patients who had HPV positivity, had no expression of $\mathrm{p} 16$, we can explain this result as a prediction of progression of the cancer, since the absence of p16 expression is related to development of neoplasm., and it needs to be correlated with stages of tumors and outcomes of treatment (Makitie, MacMillan et al. 2003).

Previous studies showed that aberrant silencing is highly associated with altered cell cycle regulation during carcinogenesis, specially silencing of CDKN2A which encodes the p16 protein [30]. Our results showed $50 \%$ of patients who had HPVhr 16 positivity, had positive expression also of p16 antibody, This result agreed with many previous studies [31-33].

After using chi-square test we found that there is positive correlation between HPV (expression) and P16 (expression) with $\mathrm{P}$-Value $=0.001$, Chi-Square $=13.105$, so we tested the power of 
relationship by using Spearman test, and we found positive medium relationship with $\{\mathrm{P}=0.000>(\alpha=0.05), \mathrm{r}=0.378\}$, this result is compatible with many previous studies showed an established association between HPV positivity and p16 overexpression [33], sowe suggest a potential role of p16 in investigating HPVhr 16 in nasopharyngeal carcinoma.

Although this result needs to be confirmed in wider studies, to investigate the prognostic role and its effect on outcomes of treatment, as it was confirmed in many studies $[12,34]$.

\section{Conclusion}

Expression of HPV16 was $13.5 \%$, and P16ink4a was about $15 \%$ in a sample of 89 nasopharyngeal carcinoma patients. We stated in this study that P16ink4a has a moderate correlation with HPV16 and that could nominate it to be a potential marker for the virus HPV16 in nasopharyngeal carcinoma, but further studies are needed to confirm this result and to use it in routine examination to predect the prognosis and treat cancer earlier.

Our results also refer to the importance of absence of p16 in most cases and that should havean increased attention specially when it is correlated with the stage of cancer and prognosis.

To the best of our knowledge, this is the first experimental report of investigating HPVhr 16 in NPCs among Syrian patients in the period between 2010-2017.

\section{References}

[1]. Jeyakumar A, Brickman TM, Doerr T. Review of nasopharyngeal carcinoma. Ear Nose Throat J. 85(3):168-70, 172-3, 84. Pubmed PMID: 16615599.

[2]. Lo KW, To KF, Huang DP (2004) Focus on nasopharyngeal carcinoma. Cancer cell.2004 May;5(5):423-8. Pubmed PMID: 15144950.

[3]. Robinson M, Suh YE, Paleri V, Devlin D, Ayaz B, Pertl L, et al. Oncogenic human papillomavirus-associated nasopharyngeal carcinoma: an observational study of correlation with ethnicity, histological subtype and outcome in a UK population. Infect Agent Cancer.2013 Aug 12;8(1):30. Pubmed PMID: 23938045.

[4]. Chaturvedi AK. Epidemiology and clinical aspects of HPV in head and neck cancers. Head neck pathol. 2012 Jul;6 Suppl 1(Suppl 1):S16-24.Pubmed PMID: 22782220.

[5]. Lo EJ, Bell D, Woo J, Li G, Hanna EY, El-Naggar AK, et al. Human papillomavirus \& WHO type I nasopharyngeal carcinoma. Laryngoscope.2010 Oct;120(10):1990-7. Pubmed PMID: 20824783.

[6]. Lin Z, Khong B, Kwok S, Cao H, West RB, Le QT, et al. Human papillomavirus 16 detected in nasopharyngeal carcinomas in white Americans but not in endemic Southern Chinese patients. Head neck. 2014 May;36(5):709-14. Pubmed PMID: 23616441

[7]. Maxwell JH, Kumar B, Feng FY, McHugh JB, Cordell KG, Eisbruch A, et al. HPV-positive/p16-positive/EBV-negative nasopharyngeal carcinoma in white North Americans. Head neck.2010 May;32(5):562-7. Pubmed PMID: 19757421.

[8]. Duray A, Descamps G, Decaestecker C, Remmelink M, Sirtaine N, Lechien J, et al. Human papillomavirus DNA strongly correlates with a poorer prognosis in oral cavity carcinoma. Laryngoscope.2012; 122(7):1558-65. Pubmed PMID: 22532307.

[9]. Walline HM, Komarck C, McHugh JB, Byrd SA, Spector ME, Hauff SJ, et al.High-risk human papillomavirus detection in oropharyngeal, nasopharyngeal, and oral cavity cancers: comparison of multiple methods. JAMA Otolaryngol Head Neck Surg.2013;139(12):1320-7. Pubmed PMID: 24177760 .

[10]. Witcher M, Emerson BM. Epigenetic silencing of the p16(INK4a) tumor suppressor is associated with loss of CTCF binding and a chromatin boundary. Mol Cell.2009;34(3):271-84. Pubmed PMID: 19450526.

[11]. Lo KW, Cheung ST, Leung SF, van Hasselt A, Tsang YS, Mak KF, et al. Hypermethylation of the p16 gene in nasopharyngeal carcinoma. Cancer research.1996;56(12):2721-5. Pubmed PMID: 8665502
[12]. Jiang W, Chamberlain PD, Garden AS, Kim BY, Ma D, Lo EJ, et al.Prognostic value of p16 expression in Epstein-Barr virus-positive nasopharyngeal carcinomas. Head neck.2016; 38 Suppl 1:E1459-66. Pubmed PMID: 26560893.

[13]. Makitie AA, MacMillan C, Ho J, Shi W, Lee A, O'Sullivan B, et al. Loss of p16 expression has prognostic significance in human nasopharyngeal carcinoma. Clin Cancer Res.2003;9(6):2177-84. Pubmed PMID: 12796384.

[14]. Gronhoj Larsen C, Gyldenlove M, Jensen DH, Therkildsen MH, Kiss K, Norrild B, et al.Correlation between human papillomavirus and p16 overexpression in oropharyngeal tumours: a systematic review. Br J Cancer.2014;110(6):1587-94. Pubmed PMID: 24518594.

[15]. El-Naggar AK, Westra WH.p16 expression as a surrogate marker for HPVrelated oropharyngeal carcinoma: a guide for interpretative relevance and consistency. Headneck.2012;34(4):459-61. Pubmed PMID: 22180304.

[16]. Luo S, Zhao L, Wang J, Xu M, Li J, Zhou B, et al. Clinical outcomes for early-stage nasopharyngeal carcinoma with predominantly WHO II histology treated by intensity-modulated radiation therapy with or without chemotherapy in nonendemic region of China. Head neck.2014;36(6):841-7. Pubmed PMID: 23720240.

[17]. Cregger M, Berger AJ, Rimm DL. Immunohistochemistry and quantitative analysis of protein expression. Arch Pathol Lab Med.2006;130(7):1026-30. Pubmed PMID: 16831029.

[18]. Matos LL, Stabenow E, Tavares MR, Ferraz AR, Capelozzi VL, Pinhal MA. Immunohistochemistry quantification by a digital computer-assisted method compared to semiquantitative analysis. Clinics (Sao Paulo).2006;61(5):417-24. Pubmed PMID: 17072439.

[19]. Han CP, Lee MY, Tzeng SL, Yao CC, Wang PH, Cheng YW, et al. Nuclear Receptor Interaction Protein (NRIP) expression assay using human tissue microarray and immunohistochemistry technology confirming nuclear localization. J Exp Clin Cancer Res.2008;27(1):25. Pubmed PMID: 18673574.

[20]. Ang KK, Harris J, Wheeler R, Weber R, Rosenthal DI, Nguyen-Tan PF, et al.Human papillomavirus and survival of patients with oropharyngeal cancer. N Engl J Med.2010;363(1):24-35. Pubmed PMID: 20530316.

[21]. Gillison ML, Koch WM, Capone RB, Spafford M, Westra WH, Wu L, et al. Evidence for a causal association between human papillomavirus and a subset of head and neck cancers. J Natl Cancer Inst.2000;92(9):709-20. Pubmed PMID: 10793107.

[22]. Stenmark MH, McHugh JB, Schipper M, Walline HM, Komarck C, Feng FY, et al () Nonendemic HPV-positive nasopharyngeal carcinoma: association with poor prognosis. Int J Radiat Oncol Biol Phys.2014;88(3):580-8. Pubmed PMID: 24521676.

[23]. Bonomi M, Abbott RW, Patsias A, Misiukiewicz K, Demicco EG, Zhang $\mathrm{D}$, et al. Role of human papillomavirus and p16 staining in a patient with head and neck cancer presenting with a synchronous lung nodule: a case report and review of the literature. J Clin Oncol.2015;33(3):e13-5. Pubmed PMID: 24590649.

[24]. Gulley ML, Nicholls JM, Schneider BG, Amin MB, Ro JY, Geradts J. Nasopharyngeal carcinomas frequently lack the p16/MTS1 tumor suppressor protein but consistently express the retinoblastoma gene product. Am J Pathol.1998;152(4):865-9. Pubmed PMID: 9546345.

[25]. Baba Y, Tsukuda M, Mochimatsu I, Furukawa S, Kagata H, Satake K, et al. Reduced expression of p16 and p27 proteins in nasopharyngeal carcinoma. Cancer Detect Prev.2001;25(5):414-9. Pubmed PMID: 11718447.

[26]. Olshan AF, Weissler MC, Pei H, Conway K, Anderson S, Fried DB, et al.Alterations of the $\mathrm{p} 16$ gene in head and neck cancer: frequency and association with p53, PRAD-1 and HPV. Oncogene.1997;14(7):811-8. Pubmed PMID: 9047388.

[27]. Reed AL, Califano J, Cairns P, Westra WH, Jones RM, Koch W, et al. High frequency of p16 (CDKN2/MTS-1/INK4A) inactivation in head and neck squamous cell carcinoma. Cancer Res.1996;56(16):3630-3. Pubmed PMID: 8705996.

[28]. Wadsworth B, Bumpous JM, Martin AW, Nowacki MR, Jenson AB, Farghaly H. Expression of p16 in sinonasal undifferentiated carcinoma (SNUC) without associated human papillomavirus (HPV). Head neck pathol.2011;5(4):349-54. Pubmed PMID: 21805120.

[29]. Veganzones S, Maestro ML, Rafael S, de la Orden V, Vidaurreta M, Mediero B, et al.Combined methylation of p16 and hMLH1 (CMETH2) discriminates a subpopulation with better prognosis in colorectal cancer patients with microsatellite instability tumors. Tumour biol.2015;36(5):3853-61. Pubmed PMID: 25576161.

[30]. Zhao R, Choi BY, Lee MH, Bode AM, Dong Z. Implications of Genetic and Epigenetic Alterations of CDKN2A (p16(INK4a)) in Cancer. EBioMedicine.2016;8:30-9. Pubmed PMID: 27428416.

[31]. Doxtader EE, Katzenstein A-LA.The relationship between p16 expression and high-risk human papillomavirus infection in squamous cell carcinomas from sites other than uterine cervix: a study of 137 cases. Hum 
pathol.2012;43(3):327-32. Pubmed PMID: 21840041.

[32]. Santos M, Landolfi S, Olivella A, Lloveras B, Klaustermeier J, Suárez H, et al.p16 overexpression identifies HPV-positive vulvar squamous cell carcinomas. Am J Surg Pathol.2006;30(11):1347-56. Pubmed PMID: 17063073.

[33]. König F, Krekeler G, Hoenig JF, Cordon-Cardo C, Fischer G, Korabiowska M. Relation between human papillomavirus positivity and p16 expres- sion in head and neck carcinomas-a tissue microarray study. Anticancer Res.2007;27(1A):283-8. Pubmed PMID: 17352245.

[34]. Wittekindt C, Wagner S, Sharma SJ, Wurdemann N, Knuth J, Reder $\mathrm{H}$, et al. HPV - A different view on Head and Neck Cancer. Laryngorhinootologie.2018;97(S 01):S48-S113. Pubmed PMID: 29905354. 